\title{
Effect of Variable Injection Sites for Indocyanine Green Dye on the Success of Lymphaticovenular Anastomosis
}

\author{
Takashi Nuri, MD ${ }^{1}$ Hiroyuki Iwanaga, MD ${ }^{1} \quad$ Yuki Otsuki, MD ${ }^{1} \quad$ Koichi Ueda, MD \\ ${ }^{1}$ Department of Plastic and Reconstructive Surgery, Osaka Medical \\ Address for correspondence Takashi Nuri, MD, Department of Plastic \\ and Reconstructive Surgery, Osaka Medical College, 2-7, Daigaku-cho, \\ Takatsuki, Osaka, 569-8686, Japan (e-mail: pla042@osaka-med.ac.jp). \\ J Reconstr Microsurg Open 2019;4:e92-e95.
}

\begin{abstract}
Background Indocyanine green lymphography (ICGL) allows more accurate visualization for lymphaticovenular anastomosis (LVA). However, the protocol for ICGL has not been established yet. We investigated how injection sites of ICG affect lymphography results by comparing ICGL images based on different injection sites on the same patients.

Methods Our hospital followed two ICGL protocols over time: ICG was injected into patients' 1st to 4th toe web spaces during 2013 to 2017 (Protocol 1), but into their lateral and medial ankles and 1st and 4th toe web spaces starting in 2018 (Protocol 2). Ten patients with secondary lymphedema who underwent LVA twice, using each protocol, were included in this study. We compared their results in detail and evaluated the effects of variable ICG injection sites.

Results The average period between patients' first and second LVAs was 506 days. In six patients, Protocol 2 detected new and additional linear findings that had not been detected by Protocol 1. Average reduction of lower limb circumferences after second LVAs (using Protocol 2) was $2.73 \mathrm{~cm}$ in patients who showed new linear findings,

\section{Keywords}

- indocyanine green lymphography

- injection site whereas those with no new findings showed little reduction.

Conclusion LVA based on ICG injections only into the dorsum of the foot can miss valuable findings. Variable ICG injection sites may improve detection of lymphatic flow and LVA efficacy.
\end{abstract}

Lymphangiography using indocyanine green (ICG) dye makes it possible to visualize lymph vessels in real time, and is an important tool for successful lymphaticovenular anastomoses (LVAs). ${ }^{1,2}$ However, protocols for ICG lymphography (ICGL) including injection sites have not been established yet. Radionuclide lymphoscintigraphy (RNL) has been widely used to diagnose lower limb lymphedema. In the standard RNL protocol, a small volume of calibrated nanocolloids labeled with $99 \mathrm{~m}$ is injected into the first web space of each foot. ${ }^{3}$ Usually, ICGL is also performed after the webspace injections. However, whether injections into the toe web spaces or dorsum of the foot can accurately detect the lymph dynamics in lymphedema patients is unclear.

received

July 7, 2019

accepted after revision

September 29, 2019
DOI https://doi.org/

$10.1055 / \mathrm{s}-0039-3400245$

ISSN 2377-0813.
Although several recent anatomical studies of lymph vessels using cadaver dissection showed normal lymph systems, ${ }^{4,5}$ the smooth muscle function of lymph vessels is key in evaluating the drainage effect of LVA. Therefore, examining living patients' remaining smooth muscle function and analyzing how lymphatic dynamics have changed due to lymphatic obstruction can reveal vital information.

In our hospital, ICGL had been performed using subdermal injections into toe web spaces. Since January 2018, this protocol was changed to include not only the toe web spaces but also injections into the medial and lateral ankles. We have had several patients with secondary

Copyright @ 2019 by Thieme Medical Publishers, Inc., 333 Seventh Avenue, New York, NY 10001, USA. Tel: +1(212) 584-4662.
License terms

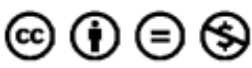


Effect of Variable Injection Sites for Indocyanine Green Dye Nuri et al. e93

\begin{tabular}{|c|c|c|c|c|c|c|c|c|c|c|}
\hline 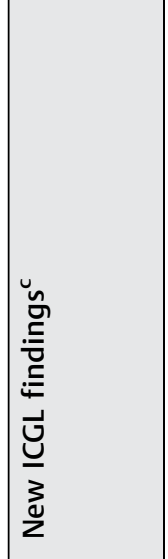 & 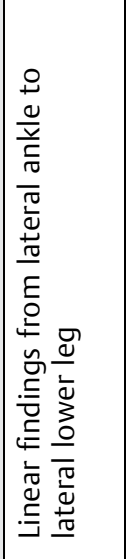 & 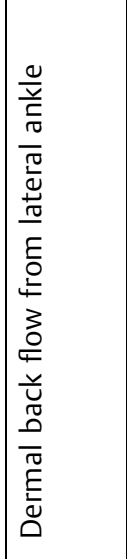 & 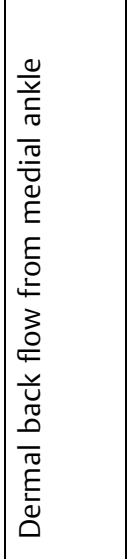 & 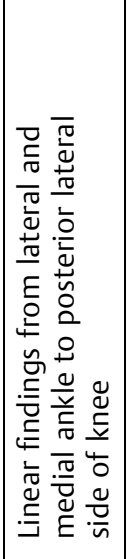 & 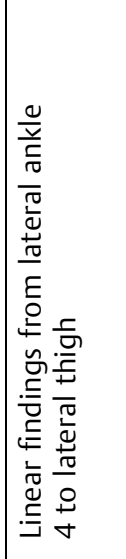 & 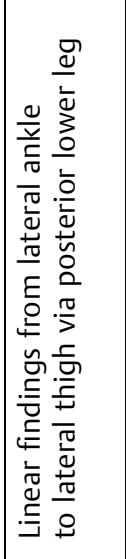 & 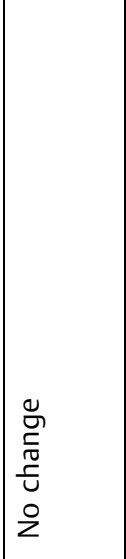 & 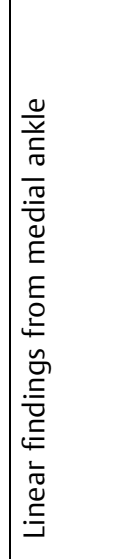 & 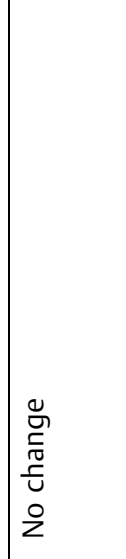 & 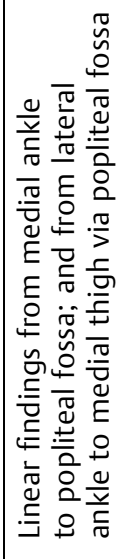 \\
\hline 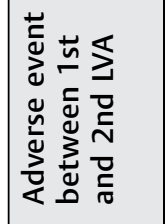 & $\mid \begin{array}{l}0 \\
\tilde{0} \\
z\end{array}$ & $\begin{array}{l}0 \\
\text { ¿े } \\
z\end{array}$ & $\begin{array}{l}\tilde{\Sigma} \\
\tilde{\Sigma}\end{array}$ & 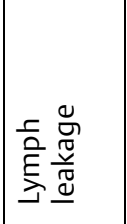 & $\mid \frac{\underline{\frac{n}{3}}}{\overline{\bar{\Xi}}}$ & $\frac{\underline{\frac{n}{E}}}{\overline{\bar{U}}}$ & $\begin{array}{l}0 \\
\tilde{0} \\
z\end{array}$ & $\begin{array}{l}0 \\
\tilde{o} \\
z\end{array}$ & $\begin{array}{l}0 \\
\tilde{L} \\
z\end{array}$ & $\mid \begin{array}{l}0 \\
\tilde{0} \\
z\end{array}$ \\
\hline 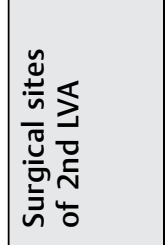 & 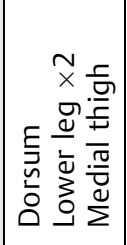 & 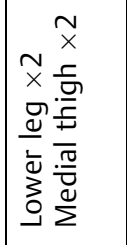 & 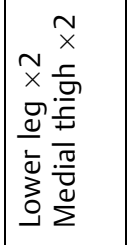 & 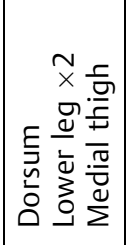 & 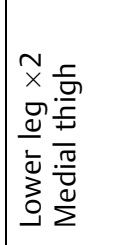 & 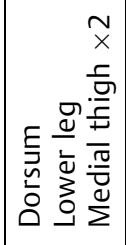 & 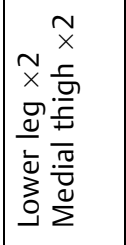 & 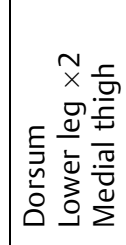 & 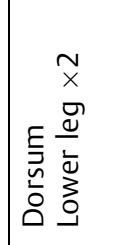 & 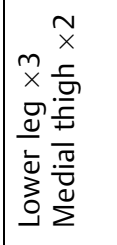 \\
\hline 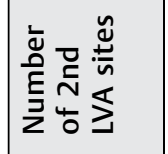 & $\nabla$ & $\nabla$ & $\nabla$ & $\nabla$ & $m$ & $\nabla$ & $\nabla$ & $\nabla$ & $m$ & in \\
\hline 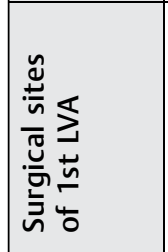 & 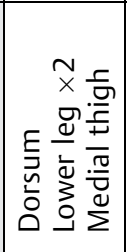 & 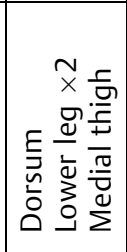 & 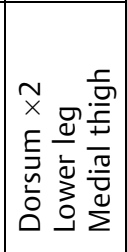 & 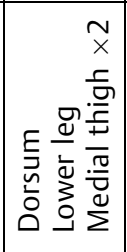 & 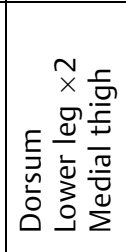 & 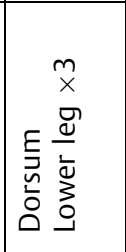 & 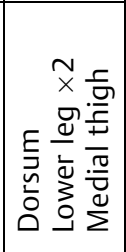 & 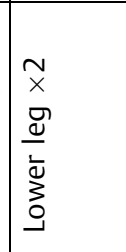 & 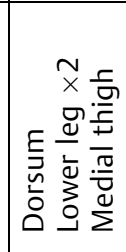 & 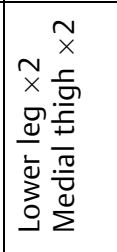 \\
\hline 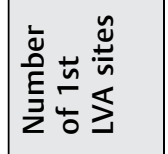 & $\nabla$ & $\nabla$ & $\nabla$ & $\nabla$ & $\nabla$ & $\nabla$ & $\nabla$ & $\sim$ & $\nabla$ & $\nabla$ \\
\hline 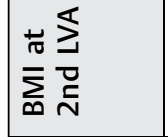 & $\begin{array}{l}\infty \\
\stackrel{\infty}{\infty} \\
\sim\end{array}$ & 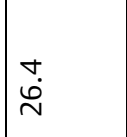 & $\begin{array}{l}\stackrel{0}{\dot{\omega}} \\
\sim\end{array}$ & $\begin{array}{l}\infty \\
\stackrel{\sim}{\sim} \\
\dot{\sim}\end{array}$ & $\begin{array}{l}\text { Ln } \\
\sigma \\
\sigma\end{array}$ & $\begin{array}{l}\stackrel{\sigma}{\sim} \\
\stackrel{\sim}{\sim}\end{array}$ & $\stackrel{m}{\stackrel{n}{v}}$ & $\begin{array}{l}\dot{\varphi} \\
\stackrel{\leftrightarrow}{m}\end{array}$ & $\stackrel{\infty}{\grave{N}}$ & ஸे. \\
\hline 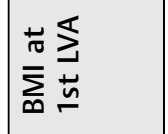 & 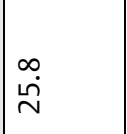 & $\stackrel{i}{\grave{n}}$ & $\stackrel{\sim}{\stackrel{N}{N}}$ & 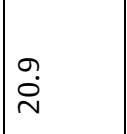 & $\stackrel{\nabla}{\sigma}$ & $\begin{array}{l}0 \\
\ddot{N}\end{array}$ & $\stackrel{9}{\sigma}$ & $\overline{\dot{m}}$ & $\stackrel{\sim}{\grave{N}}$ & $\stackrel{\sim}{\underset{N}{\sim}}$ \\
\hline 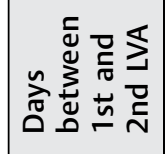 & 官 & ભે & $\stackrel{\substack{\infty \\
m}}{2}$ & $\stackrel{\text { }}{\stackrel{N}{ }}$ & $\underset{\infty}{\stackrel{m}{\infty}}$ & 合 & $\bar{\gamma}$ & 守 & $\begin{array}{l}\infty \\
0 \\
0\end{array}$ & N \\
\hline 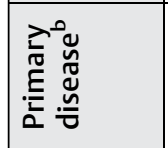 & 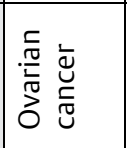 & 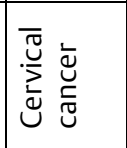 & 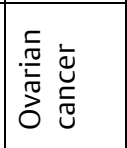 & 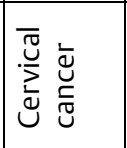 & 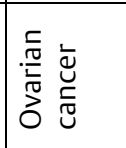 & 莺 & 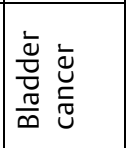 & 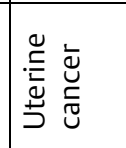 & 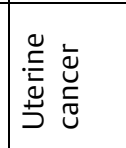 & 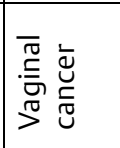 \\
\hline 芩 & $\stackrel{\circ}{\gtrless}$ & $\stackrel{m}{r}$ & $\mathscr{6}$ & in & $\stackrel{*}{\sim}$ & $\infty$ & $\hat{6}$ & 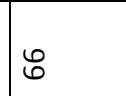 & 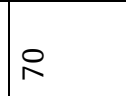 & 6 \\
\hline $\begin{array}{l}\stackrel{\mathscr{n}}{0} \\
\text { }\end{array}$ & - & $\sim$ & $m$ & $\nabla$ & in & 0 & $r$ & $\infty$ & $a$ & $\stackrel{\circ}{\circ}$ \\
\hline
\end{tabular}

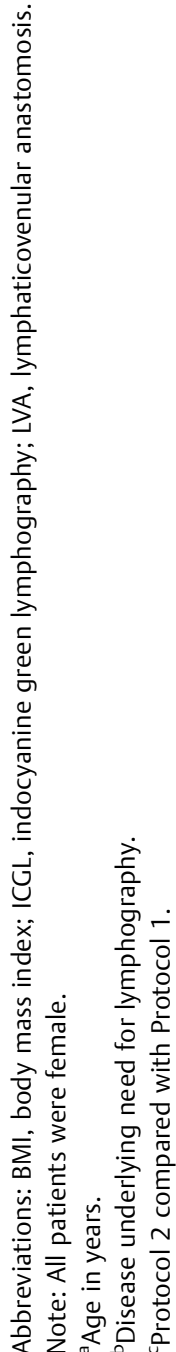


New descriptions using protocol 2

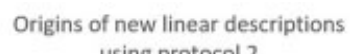
using protocol 2
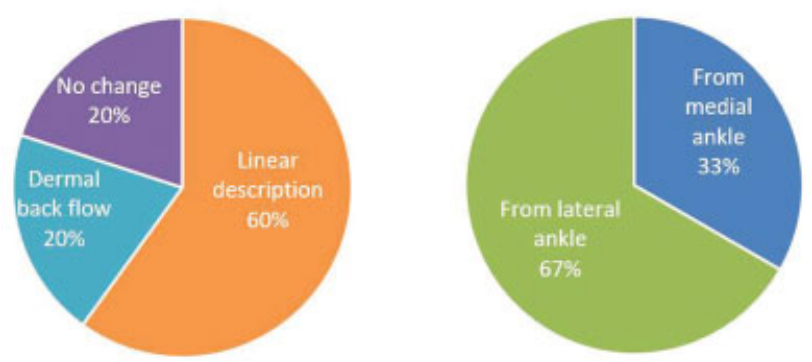

Fig. 1 Protocol 2 for indocyanine green (ICG) injection generated was observed in $80 \%$ of patients and new linear descriptions were observed in $60 \%$ of patients. Sixty percent of linear descriptions were observed from injection site of lateral ankle.

lymphedema who underwent two LVAs, which allowed us to use both the old and the new protocols on the same patients.

This study analyzed how ICG injection sites help identify suitable lymph vessels for LVA by comparing results in the same patients, using different protocols.

\section{Methods}

\section{Indocyanine Green Lymphography Examinations}

Use of ICGL to diagnose lymphedema patients was approved by the Ethical Committee of Osaka Medical College (No. 594).

\section{Protocol 1 (Old)}

From 2013 to December 2017, ICGL for lower limb had been performed using four injections of $0.2 \mathrm{~mL}(0.5 \mathrm{mg})$ ICG into the first to the fourth toe web spaces.

\section{Protocol 2 (New)}

Since January 2018, ICGL has been performed using four injections of $0.2 \mathrm{~mL}(0.5 \mathrm{mg})$ ICG into the lateral and medial ankles, and into the first and fourth toe web spaces.

\section{Lymphaticovenular Anastomosis}

We performed LVA under general anesthesia. After ICG injection, the paths of lymph vessels were observed using an infrared camera (Photodynamic-neo Hamamatsu Photonics, K.K. Hamamatsu, Japan) and the skin was marked using an oil-based pen. Subdermal veins were then evaluated using ultrasonography. Usually, four surgical sites were identified according to these findings and the condition of the edema.

\section{Patients}

Between January 2013 to November 2018, LVAs were performed on 183 patients with lower lymphedema at Osaka Medical College Hospital. Among these patients, we included in this study those who underwent LVAs twice, using Protocol 1 in their first operation and using Protocol 2 in their second operation.

For each patient, we compared the two maps of lymphatic vessels recorded after ICG injections, using Protocols 1 and 2, and evaluated the two protocols by comparing lymph vessels that were invisible in Protocol 1 but detected using Protocol 2 . The effect of LVA was evaluated by measuring the circumferences of the ankle, center of crus, knee, and thigh.

\section{Results}

We included 10 patients with secondary lymphedema (10 women) in this study (-Table $\mathbf{1}$ ). The average period between their first and second LVA was 506 days (range: 203-1,097 days). Of the 10 patients, 2 showed no clear differences between the two protocols. However, Protocol 2 produced new linear descriptions in 6 patients that were not observed using Protocol 1, including new linear descriptions from the lateral ankle from 5 patients (-Figs. 1 and 2). Among them, linear descriptions that directly include the lateral side of the thigh were observed in 2 patients, and that ascended to the medial thigh via posterior of the lower leg in 1 patient (-Fig. 3). In 3 patients, linear descriptions were observed from their medial ankle injection sites. In 2 atypical patients,

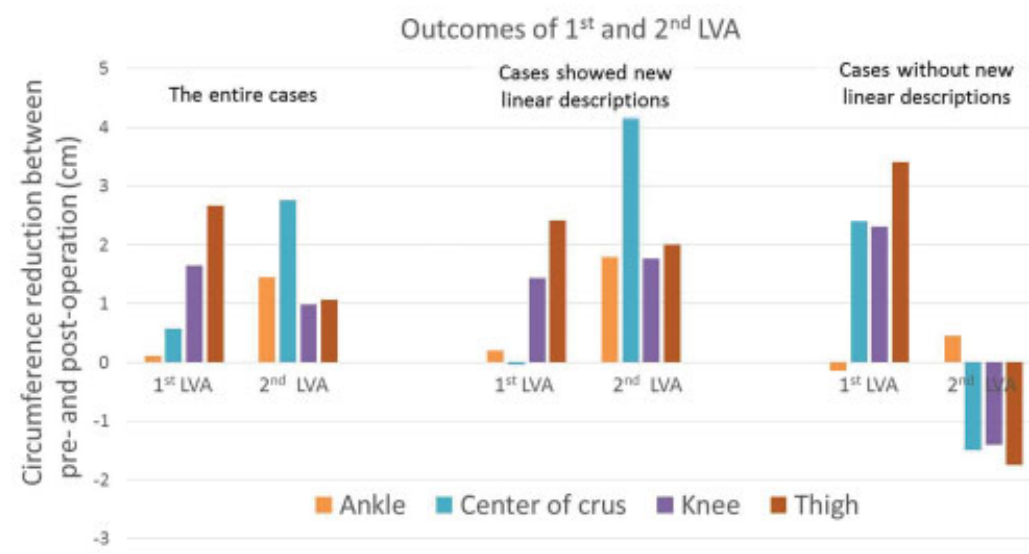

Fig. 2 Circumferences of patients' lower legs were measured at ankle, center of the crus, knee, and thigh. At 6 months after the second LVA using Protocol 2, average circumference reductions (in $\mathrm{cm}$ ) were ankle: 1.78 (range: $0.5-2.4$ ), center of the crus: 4.18 (1.3-6.8), knee: 1.76 (0.5-5.9), and thigh: 2.00 ( -0.4 to 4.2 ) among patients who showed new linear descriptions, but $0.45(-2.5$ to 2.4$),-1.50(-3$ to 3$),-1.47$ $(-1.4$ to 0.5$)$, and $-1.75(-3.2$ to -0.3$)$, respectively, in patients with no new linear descriptions. 


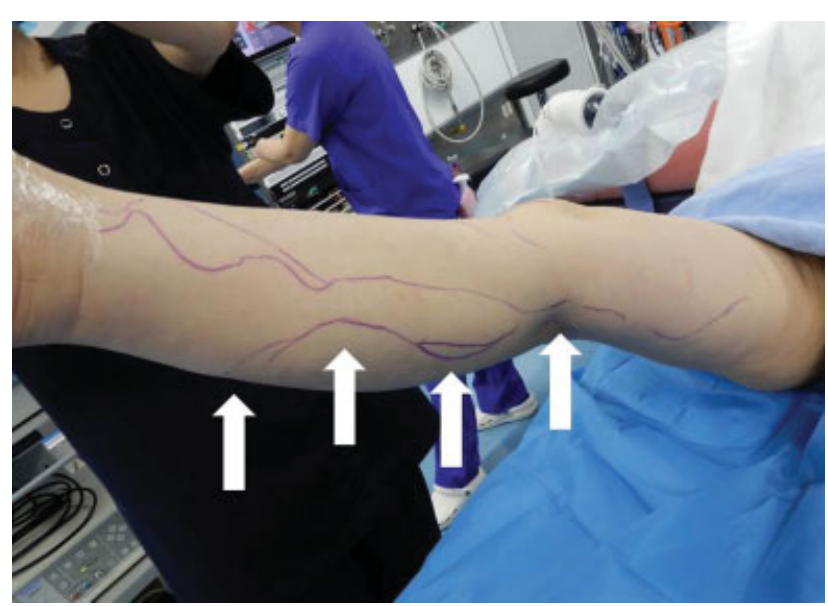

Fig. 3 The affected limb of patient 10. Lymph vessel paths were drawn using an oil-based marker. A linear description from lateral ankle passed across the lymph territory and reached the medial thigh.

the linear descriptions from the medial ankle flowed in a horizontal direction to the midline of the ankle, and then reached the medial thigh via the anterior of the lower leg.

At 6 months after the second LVA using Protocol 2, average circumference reductions (in $\mathrm{cm}$ ) were ankle: 1.78 (range: 0.5$2.4)$, center of crus: 4.18 (1.3-6.8), knee: 1.76 (0.5-5.9), and thigh: 2.00 ( -0.4 to 4.2 ) among patients who showed new linear descriptions, but 0.45 ( -2.5 to 2.4$),-1.50$ ( -3 to 3 ), -1.47 ( -1.4 to 0.5 ), and -1.75 ( -3.2 to -0.3 ), respectively, in patients with no new linear descriptions (-Fig. 3 ).

\section{Discussion}

Recent anatomical studies have clarified the paths of normal lymph systems in the lower limbs. ${ }^{4,5}$ The lymph vessels from the dorsum and the medial ankle run axially toward the anterior of the lower leg, and then ascend to the medial side of the thigh toward the inguinal lymph nodes. These paths are usually parallel with the great saphenous vein. The lymph vessels from the lateral ankle run along the lesser saphenous vein toward the popliteal lymph nodes. In our study, $67 \%$ of the new linear descriptions were from lateral ankle, which indicates that injections into the dorsum alone cannot catch up to the lateral lymph paths. In 2017, Suami developed a lymphosome concept for a normal body. ${ }^{5}$ Lymphatic territories in the lower leg were divided into two areas, the inferior inguinal and the popliteal area. Normally, collecting lymph vessels run straight, with a few interconnections between each lymph vessel. However, our results indicate that lymph dynamics in lymphedema may be different from normal. ${ }^{6}$ In some patients, lymph travels across the usual lymph territories. Usually, the ICGL is performed by injecting a tracer into the toe web spaces to expose the anterior lymphatic path. Lymphedema is known to be progressive once it has developed and cellulitis and other inflammation will adversely affect the condition. In our cases, linear descriptions were identified even in the cases who suffered from cellulitis between first and second LVA. Although this report is limited to the 10 cases we had and further study would be necessary to confirm these findings, our results suggest that varying the injection sites -that is, the lateral and medial ankles in addition to the toe web space areas-improve visualization of lymph vessels in lymphedema patients.

\section{Funding}

None.

Conflict of Interest

None declared.

\section{References}

1 Unno N, Inuzuka K, Suzuki M, et al. Preliminary experience with a novel fluorescence lymphography using indocyanine green in patients with secondary lymphedema. J Vasc Surg 2007;45(05): 1016-1021

2 Narushima M, Yamamoto T, Ogata F, Yoshimatsu H, Mihara M, Koshima I. Indocyanine green lymphography findings in limb lymphedema. J Reconstr Microsurg 2016;32(01):72-79

3 Pecking AP, Albérini JL, Wartski M, Edeline V, Cluzan RV. Relationship between lymphoscintigraphy and clinical findings in lower limb lymphedema (LO): toward a comprehensive staging. Lymphology 2008;41(01):1-10

4 Suami H, Scaglioni MF. Anatomy of the lymphatic system and the lymphosome concept with reference to lymphedema. Semin Plast Surg 2018;32(01):5-11

5 Suami H. Lymphosome concept: anatomical study of the lymphatic system. J Surg Oncol 2017;115(01):13-17

6 Blum KS, Proulx ST, Luciani P, Leroux JC, Detmar M. Dynamics of lymphatic regeneration and flow patterns after lymph node dissection. Breast Cancer Res Treat 2013;139(01):81-86 